A N N A L E S Annales de Bretagne et des Pays de l'Ouest

\title{
La fabrique, les couvents et les académies dans le diocèse de Nicolet (1852-1904)
}

Jean Roy

\section{(2) OpenEdition}

\section{Journals}

\section{Édition électronique}

URL : http://journals.openedition.org/abpo/1339

DOI : $10.4000 /$ abpo. 1339

ISBN : 978-2-7535-1492-8

ISSN : 2108-6443

Éditeur

Presses universitaires de Rennes

Édition imprimée

Date de publication : 20 décembre 2003

Pagination : $95-106$

ISBN : 978-2-86847-933-4

ISSN : 0399-0826

\section{Référence électronique}

Jean Roy, «La fabrique, les couvents et les académies dans le diocèse de Nicolet (1852-1904) »,

Annales de Bretagne et des Pays de l'Ouest [En ligne], 110-4 | 2003, mis en ligne le 20 décembre 2005, consulté le 01 mai 2019. URL : http://journals.openedition.org/abpo/1339 ; DOI : 10.4000/abpo.1339 


\title{
La fabrique, les couvents et les académies dans le diocèse de Nicolet (1852-1904)
}

\author{
Jean Roy \\ Professeur d'Histoire contemporaine \\ Centre interuniversitaire d'études québécoises \\ Université du Québec à Trois-Rivières
}

L'échec de la loi des écoles de fabrique (1824) a jeté dans l'ombre le rôle que cette institution a pourtant continué de jouer en éducation. C'est, en effet, grâce à sa contribution financière et aux initiatives des curés des paroisses de même qu'au dynamisme des congrégations religieuses enseignantes que les villages du Québec se sont enrichis de couvents et d'académies dans la seconde moitié du $19^{\mathrm{e}}$ siècle $^{1}$. Toutefois ceci n'aurait pu se faire sans la mise en place par l'assemblée du Bas-Canada d'une législation étatique qui favorisait de nouvelles conditions matérielles pour les communautés religieuses ni sans la volonté manifeste de l'Église de s'emparer de l'enseignement public.

Mon objectif est de démontrer comment ceci s'est articulé. Je le ferai d'abord en balisant les conditions nationales des implantations, pour examiner ensuite les faits locaux qui ont permis l'établissement des congrégations féminines et masculines dans les paroisses du diocèse de Nicolet, entre 1852 et 1904.

\section{Les facteurs externes des implantations}

L'action de ces congrégations répondait à une stratégie de l'Église qui visait à exercer un meilleur contrôle de l'instruction publique. Elle se sen-

1. Couvent et académie (collège) ont ici des acceptations très larges car ils désignent à la fois la maison d'école et l'habitation d'une communauté de sœurs ou de frères. École de village ou pensionnat-école, l'institution était financée par l'État, la commission scolaire locale qui prélevait une taxe sur les propriétaires, la rétribution mensuelle des parents et les revenus du pensionnat. Ceux-ci comptaient pour beaucoup dans le maintien des religieux. L'importante révision du programme d'enseignement dans les classes élémentaires publiques apportée en 1888, répartissait les huit années de la scolarité en école élémentaire, école modèle et école académique, chacune comptant respectivement 4, 2 et 2 années. Pour toutes ces questions, deux ouvrages : Dufour, Andrée, Histoire de l'éducation au Québec, Montréal, Boréal, 1997, 126 p., et CHARLAND, Jean-Pierre, L'entreprise éducative au Québec, 1840-1900, Québec, Presses de l'Université Laval, 2000, 452 p. 
tait d'autant plus à l'aise qu'elle n'avait qu'à s'appuyer sur la législation scolaire et le droit foncier et immobilier qui régissait la propriété ecclésiastique. La loi de 1824 autorisait les fabriques, avec l'accord de l'évêque, à créer des écoles et à les financer en recourant au quart de leurs revenus. Grâce à l'amendement apporté en 1827, elles étaient en mesure "d'acquérir un emplacement d'un arpent carré avec les bâtisses nécessaires ${ }^{2}$ ". En l'espace de 5 ans, écrit Lucien Lemieux ${ }^{3}, 68$ écoles furent établies en autant de paroisses, administrées comme des écoles privées et financées à travers les fabriques. Néanmoins, c'était bien insuffisant. Si bien qu'en 1829, le gouvernement colonial institua l'École des syndics qu'il plaça sous l'autorité de la Chambre d'assemblée. Il lui octroya des subventions pour sa diffusion, ce qui lui assura un succès indéniable. Mais, en 1836, pour des raisons politiques, il mit fin à son aide financière. L'évêque de Québec, $M^{\text {gr }}$ Signaï, en prit acte et, tout en disant souhaiter que la situation se rétablisse rapidement, rappela aux curés la possibilité qu'avaient les fabriques de soutenir financièrement les écoles. Il les incita à convaincre les marguilliers d'agir dans ce sens sans autre autorisation de sa part ${ }^{4}$. En 1841, le gouvernement donna une nouvelle impulsion à l'éducation par la loi de l'Instruction publique. C'est sous son empire que se place l'activisme du clergé, invité par l'évêque de Québec à occuper un des cinq postes de commissaire ${ }^{5}$.

L'Église pouvait alors penser prendre les moyens utiles pour atteindre ses objectifs. L'établissement des congrégations religieuses dans les villages en était un. En 1851, les membres de la commission d'études qui préparaient le premier concile provincial de Québec trouvaient avantageux de multiplier les écoles tenues par les religieuses, notamment pour assurer une relève des enseignantes. Dix ans plus tard, l'évêque Bourget franchit une autre étape lorsqu'il s'adressa à son homologue de Québec, Turgeon. Il lui exprima alors sa crainte que « l'État ne favorise l'enseignement laïc et peut-être mixte ${ }^{6}$ ». En conséquence, il demanda que le clergé s'empare des écoles de villages.

Pour y parvenir l'Église mit à profit des articles de la législation du gouvernement du Bas-Canada sur la propriété. Ainsi, celui-ci avait redonné aux fabriques et aux communautés religieuses le droit d'acquérir des biens meubles et immeubles pour des fins éducatives. L'acte de 1830, précisé par

2. Pagnuelo, S., Études historiques et légales sur la liberté religieuse en Canada, Montréal, Beauchemin et Valois. 1872, p. 199.

3. VoIsINE, Nive (dir.), Histoire du catholicisme québécois. Les $18^{e}$ et 19 e siècles, tome I, Les années difficiles (1760-1839), par Lucien LEMIEUX, Montréal, Boréal, 1984, p. 190.

4. " Circulaire à messieurs les curés du diocèse, au sujet de l'éducation élémentaire dans la province ", Québec, 2 mai 1836. Dans Mandements des évêques de Québec publiés par $\mathrm{M}^{\mathrm{gr}} \mathrm{H}$. TÊTu et l'abbé C. O. GAGNON, Québec, Imprimerie générale A. Côté et C $\mathrm{C}^{\mathrm{ie}}, 1888$, Vol. 3, p. 341-342.

5. "Circulaire pour engager les curés à favoriser la mise à exécution du Bill des écoles, et à accepter la charge de commissaire à cette fin ", Québec, 30 décembre 1841, ibid., p. 407-409.

6. Volsine, Nive et WARD, Yvonne, Histoire des Soeurs de la Charité, tome 1, L'âme de la fondation, Marcelle Mallet, Beauport, MNH, 1998, p. 186 et 129. 
celui du 19 mars 1839, leur reconnaissait, en effet, les acquis immobiliers pourvu qu'ils soient enregistrés au plus tard le 19 mars 1841. De la sorte, les fabriques et les congrégations religieuses recouvraient pleinement la liberté perdue en 1743, d'acquérir des biens pour des fins de culte et d'instruction $^{7}$. La congrégation des Sœurs de l'Assomption, appelée à devenir la plus importante communauté du diocèse de Nicolet, eut à s'en prévaloir avant de se répandre dans les paroisses.

\section{L'essaimage des congrégations de femmes : les Sœurs de l'Assomption}

En 1852, date de sa création, le diocèse de Trois-Rivières n'avait que deux seules communautés enseignantes sur son territoire : les Frères des Écoles chrétiennes et les Ursulines. Toutes deux étaient fixées à TroisRivières. La première tenait une académie depuis 1844, alors que les filles de Marie de l'Incarnation possédaient un couvent et un externat depuis leur arrivée en 1697. C'était peu. Par ailleurs limité aux paroisses de la rive nord du diocèse si on excepte le séminaire-collège de Nicolet. Or, l'époque était au recrutement d'instituts en Europe et à des créations canadiennes. L'illustrent, en 1853, l'émigration des Sœurs de la Présentation de Marie et leur installation à Saint-Hyacinthe, et la fondation des Sœurs de l'Assomption de la Sainte-Vierge à Saint-Grégoire.

La chronologie de leur essaimage les montre d'abord dans les unités paroissiales les plus rapprochées de Saint-Grégoire, le lieu de leur fondation. Poursuivant leur expansion, elles occupaient, en 1904, 15 des 21 couvents du diocèse ${ }^{8}$ (cf. figure). Autant de lieux, autant de projets. Mais, il n'est pas d'implantation sans péripéties, ce que démontrent les établissements de Baie-du-Febvre et de Nicolet.

Dès 1856, les Sœurs de l'Assomption avaient songé à demander l'incorporation civile ${ }^{9}$. Cela ne se fit pas. Leur fondateur, le curé Jean Harper continua, à titre personnel, non sans mêler ses finances à celles de la fabrique et à celles des religieuses, à les gérer. Ces conditions n'étaient pas saines. De plus, une expansion était à envisager. Autant de raisons qui militaient en faveur d'une reconnaissance civile de la communauté et de la liberté d'action qui l'accompagnait. La démarche aboutit en 1865, l'année de leur déplacement à Baie-du-Febvre, leur première mission. La voie était ainsi ouverte à l'appropriation. Des faits antérieurs l'avaient préparée.

7. Bien que capitale, cette législation a peu retenu l'attention des historiens, si ce n'est celles de Boucher, Denis, La loi des fabriques du Québec, Université Saint-Paul, Ottawa, 1968, 371 p., et Dussault, Gabriel de, avec la collaboration de MARTEL, Gilles, Charisme et économie. Les cinq premières communautés masculines établies au Québec sous le régime anglais, Département de Sociologie, Université Laval, 1981, 149 p.

8. Le diocèse comptait alors 62 paroisses.

9. LESAGE, Germain, Les origines des Soeurs de l'Assomption de la Sainte Vierge. Nicolet, Éditions ASV, 1957, 342 p., (re 281); du même, Le Transfert à Nicolet des Sœurs de l'Assomption de la Sainte-Vierge, 1854-1874, Nicolet, Éditions A.S.V., 1965, 323 p. 
Les couvents et académies (collèges) du diocèse de Nicolet en 1904

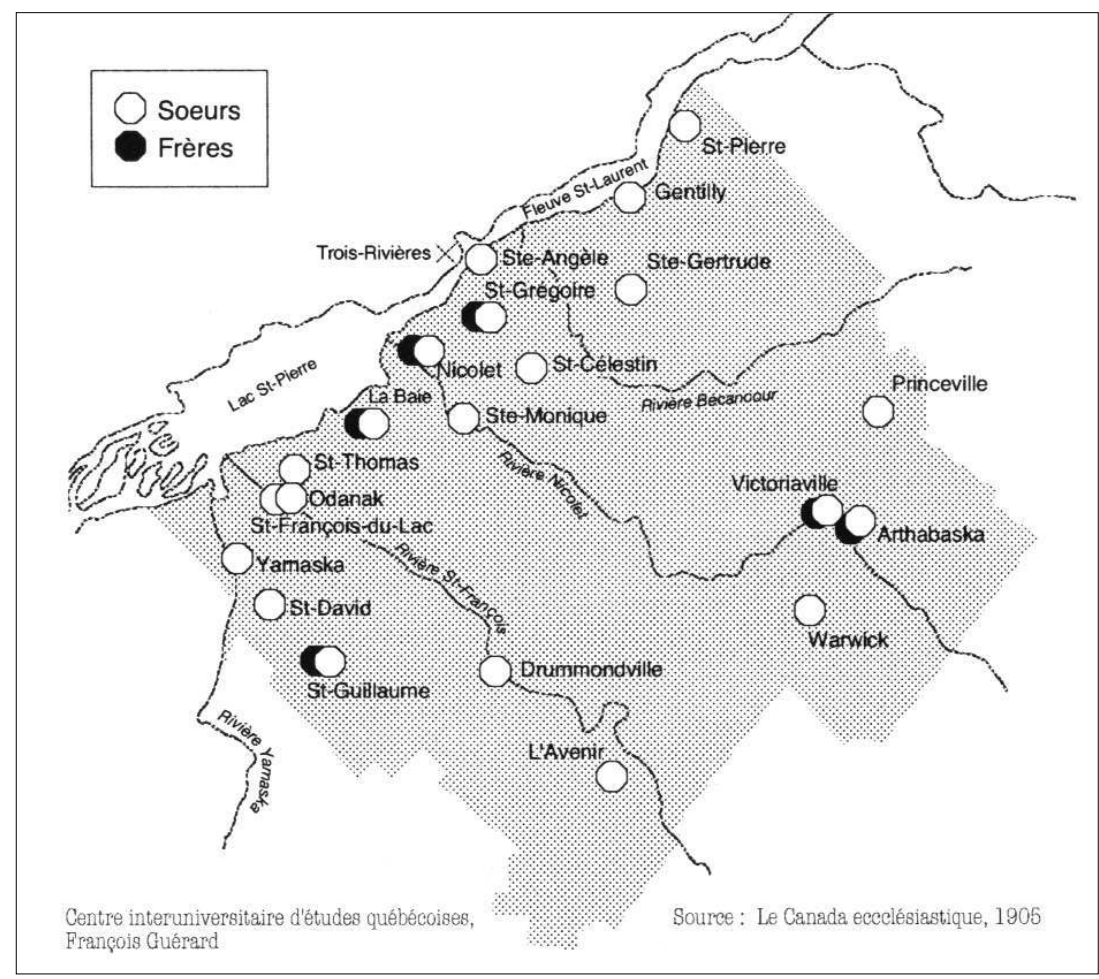

Le 11 novembre 1858, le curé de Baie-du-Febvre n'était pas loin de la panique lorsqu'il écrivit à son évêque pour lui faire connaître les derniers développements au sujet de "l'affaire de la maison de la fabrique». Cette maison et son terrain avaient fait l'objet d'un legs testamentaire de la part du curé Charles V. Fournier, le 6 mars 1835. En 1839, l'école de fabrique était transférée dans ce que l'on convenait d'appeler " la maison Fournier ». Le rez-de-chaussée servait à l'enseignement des garçons et l'instituteur, responsable de l'école entière, engageait une maîtresse pour éduquer les filles qui prenaient place l'étage. Le curé de la paroisse, appelé plus tard à occuper le poste de président de la commission scolaire, assurait la direction de l'école et pourvoyait à son entretien. Il n'existe cependant aucune trace qu'il ait puisé à même les revenus de la fabrique, ainsi qu'il en avait été préalablement autorisé ${ }^{10}$.

C'est beaucoup d'années plus tard que le curé Carrier reprit la question du financement de l'école de fabrique devenue école publique. Le bruyant

10. Bellemare, Joseph-Elzéar, Histoire de la Baie-Saint-Antoine dite Baie-du-Febvre, 16831911, Montréal, Imprimerie La Patrie, 1911, p. 487-525. Ouvrage précieux qui repose sur l'exploitation des archives paroissiales. 
mouvement de protestation contre la taxation imposée aux propriétaires, en 1846, s'était calmé et en 1853 la mesure de taxation s'appliquait. Le curé Carrier voulait savoir de son évêque, $M^{\text {gr }}$ Cooke " $s$ 'il [était] à propos d'utiliser l'argent de la fabrique pour l'établissement et le soutien des écoles. " La réponse de l'ordinaire était claire : sauf en cas de réelle nécessité et avec sa permission expresse il y avait lieu de s'en remettre aux soins prévus par le gouvernement ${ }^{11}$. Trois ans plus tard, les commissaires achetaient une maison voisine de celle de la fabrique pour l'enseignement. Avec l'accord des fabriciens, la maison Fournier servit alors de domicile à l'instituteur. Or, le bâtiment vieillissait et, en 1858, il devint urgent de le rénover. Toutefois, la facture menaçait d'être salée. Le curé se sentait coincé entre les marguilliers et les commissaires. Ceux-ci reprochaient au curé de ne pas les avoir informés de leur responsabilité financière vis-à-vis « la maison Fournier " au moment où ils avaient fait l'achat de la nouvelle maison d'école. De leur côté, les marguilliers jugeaient les coûts des travaux trop élevés pour leur budget. Seule une assemblée de la paroisse était susceptible de dénouer l'impasse. Par ailleurs, il en était pour penser qu'une injection financière importante de la part de la fabrique s'imposait pour la construction d'une école et, de la sorte, attirer une congrégation enseignante de frères ou de sœurs.

Mal à l'aise, le curé Carrier regrettait amèrement ne pas avoir carrément transmis la propriété à la commission scolaire dès 1856 et il suppliait l'évêque de lui indiquer la voie à suivre. La réponse de l'évêque Cooke arriva le 15 novembre 1858, soit quatre jours après la lettre de Carrier. Ne laissant aucun doute, il affirmait que les commissaires ne pouvaient contraindre la fabrique à défrayer les coûts des réparations. Certes, elle pouvait disposer du quart de ses revenus, mais pour aller au-delà l'assentiment de la paroisse était nécessaire. Enfin, Cooke rappelait l'obligation faite par le curé Fournier de tenir une école, sans quoi la propriété du terrain reviendrait au légataire universel, en l'occurrence l'évêque de TroisRivières. Quant à la suggestion d'une construction neuve à l'usage d'une communauté enseignante, Cooke croyait que l'idée ne " plaira jamais à la majorité ". Il lui conseilla de se reposer là-dessus et de convoquer une assemblée paroissiale, présidée par un curé voisin, si tel était le vœu. Le 13 janvier 1859, le pasteur autorisait la fabrique à défrayer les coûts des réparations de l'école, comme l'avaient demandé les paroissiens réunis en assemblée une semaine plus tôt. Il n'était fait mention ni d'un couvent ni d'une académie.

Mais l'idée faisait son chemin, devenant bientôt celle du curé Didier Paradis. Une implication financière de 6000 dollars répartis également entre lui, la fabrique et un groupe de paroissiens procura les moyens de construire un couvent sur un terrain acheté à grands frais par la fabrique en 1859. À la fin du mois d'octobre 1865, les Sœurs de l'Assomption dis-

11. Archives de l'Évêché de Trois-Rivières (AETR), Registre des lettres, vol. 1, 1852 à 1856, Cooke à Carrier, 28 décembre 1853. 
posaient « d'une jolie bâtisse en brique, à deux étages avec toit français et une cuisine attenante à la maison, également en brique ${ }^{12}$ ".

La fabrique de Nicolet n'était pas moins impliquée en éducation. Elle caressait le même projet que celles de Saint-Grégoire et de Baie-du-Febvre de compter sur une communauté enseignante. Animée vigoureusement par le curé Fortier, elle posa des gestes semblables. Elle investit dans la réparation de l'école du village, puis, associée à des paroissiens, elle acheta un terrain voisin du sien. Enfin, elle s'adressa directement à des supérieures de quelques communautés. Tôt au début du mois de juillet 1857, le marguillier " [offrait] à ces dames religieuses un établissement dans un site superbe, une ferme considérable et bocage. "L'évêque Cooke ne se montra pas opposé au projet, car il lui paraissait légitime. Mais, il voyait le grave inconvénient que posait la présence dans des villages voisins de deux couvents appartenant à des communautés distinctes pour le recrutement des pensionnaires et des postulantes à la vie religieuse. Il fit connaître son inquiétude à l'évêque d'Ottawa qui sut transmettre le message à la supérieure des Sœurs de la Charité. Cooke avait tranché en faveur des Sœurs de l'Assomption, non sans avoir demandé à leur fondateur, Charles Harper, de trouver des arrangements.

Rien ne s'accomplit dans le sens voulu par la fabrique de Nicolet, si bien que quinze années plus tard, à nouveau inspirée par le curé Fortier, elle sentit la nécessité d'entreprendre de nouvelles démarches. Une raison était que " la maison spacieuse " qu'elle comptait mettre à la disposition d'une communauté avait un urgent besoin de rénovation. Or, elle était à court de moyens financiers. En conséquence, la fabrique demanda à l'évêque la permission de " s'adresser à des communautés riches ". M $^{\text {gr }}$ Laflèche se montra d'accord pour la recherche d'une congrégation. Mais, il répondit de s'adresser aux Sœurs de l'Assomption continuant ainsi d'appliquer la politique de son prédécesseur. Sa position déplut visiblement à tous les nicolétains : fabriciens, notables et curé car on souhaitait avoir la liberté de choisir l'institut. Convaincu de la capacité des Sœurs de l'Assomption de prendre en charge cette œuvre, Laflèche ne bougea pas. Mais tel ne fut pas le cas du curé Fortier. Il tenta encore de recruter les Sœurs de la Charité d'Ottawa en leur offrant de se charger de l'assistance, ce qui était conforme à leur charisme, et de l'enseignement. La fabrique était disposée à leur octroyer un terrain pour asseoir leur couvent. Laflèche commença par donner son aval à la venue des sœurs d'Ottawa.

Puis, voilà que la supérieure de la congrégation de Saint-Grégoire vint semer le doute sur les objectifs des gens de Nicolet en affirmant que les Sœurs Grises envisageaient d'enseigner la musique, ce qui n'appartenait pas au programme élémentaire. Laflèche intervint à nouveau car il sentait qu'on ne lui avait pas tout dit. Il coupa court et écrivit à la supérieure des

12. Propos de l'annaliste des religieuses cités par Bellemare, Joseph-Elzéar, op. cit., p. 490. Dévasté par un incendie en 1883, un couvent neuf fut construit à la suite d'une souscription volontaire levée dans la paroisse. 
Sœurs de la Charité d'Ottawa pour lui dire qu'il était toujours prêt à les accueillir dans son diocèse pour leurs œuvres charitables et qu'il leur accordait la possibilité d'enseigner aux enfants du voisinage, leur reconnaissant une situation semblable à celle des Sœurs de la Providence, à Trois-Rivières. De toute évidence, la supérieure ne s'attendait pas à cela et Fortier n'anticipait pas cette réaction de son évêque. Il défendit sa position auprès de la supérieure d'Ottawa. Dans une lettre à Laflèche, la religieuse dit regretter le chagrin causé aux religieuses de Saint-Grégoire. Dans la copie qu'elle remit à Fortier, elle ajouta qu'elle s'en remettait aux décisions qui seraient prises.

Fortier n'abandonna pas car il trouvait inadmissible que Nicolet n'ait pas la congrégation de son choix. Dans un mémoire confidentiel dont le ton virulent n'est pas absent, il dénonçait les mesures discriminatoires de son évêque, lui reprochant de mal évaluer la situation. Il discréditait également les Sœurs de l'Assomption : parce qu'elles n'avaient pas de chapelain, disait-il en substance, il les trouvait mal préparées pour effectuer leurs tâches. Rempli de morgue, il les croyait " bonnes pour Saint-Célestin ", mais Nicolet n'était ni Saint-Célestin ni Baie-du-Febvre.

Il reste que le but recherché était de provoquer le déplacement de la maison-mère et du noviciat des Sœurs de l'Assomption vers Nicolet. Le premier geste de Laflèche fut d'autoriser le déplacement, puis il se ravisa. Il fit connaître sa position à Fortier l'assurant que les religieuses auraient là une école semblable à celle de Saint-Grégoire. Puis, voilà qu'au mois de juillet 1872, au grand déplaisir des paroissiens de Saint-Grégoire, il accordait à la communauté la permission de transporter la maison-mère à Nicolet. Les Sœurs Grises renoncèrent à leur mission et, au mois de novembre 1872, le curé Fortier était mandaté pour offrir les terrains aux Sœurs de l'Assomption. Un contrat de possession était passé, le 20 mai 1873, entre la fabrique et la congrégation.

Ainsi, ce fut le curé Harper qui, le premier dans le diocèse, trouva les moyens d'appliquer la politique de l'Église. Il fonda une congrégation. Puis, à ses frais, il fit aménager une maison d'enseignement pour les nouvelles religieuses. Il usa de son pouvoir sur la fabrique et de son influence sur les fidèles pour leur procurer une propriété. Ici l'événement exceptionnel est la naissance d'une communauté, car les autres faits se vérifient, sans doute avec plus ou moins d'éclat ou d'intensité, dans tous les villages.

Les legs des curés ont également joué un rôle. Les testaments des pasteurs de Baie-du-Febvre, Fournier, Paradis et Héroux, ont affiché la même et remarquable constance en faveur de l'éducation. Seul Carrier se démarqua puisqu'il gratifia principalement ses parents. À Sainte-Gertrude, Paul de Villers, fit des Sœurs de l'Assomption son principal légataire en leur donnant le terrain et les édifices qu'elles occupaient déjà. Il laissa également des sommes en faveur des institutrices afin qu'elles puissent entrer en communauté ${ }^{13}$.

13. ASN, Fabrique de Sainte-Gertrude, « Testament et Biens appartenant à Paul de Villers ». 
Bien que favorisée par l'évêque, la congrégation des Sœurs de l'Assomption ne fut pas la seule sur le territoire diocésain. Au cours de la décennie 1870, la demande fut telle que la jeune communauté était incapable d'y suffire. Lorsque d'autres communautés s'implantèrent, l'évêque protégea leur territoire et leur assigna une responsabilité partagée en les plaçant dans des villages voisins : en 1878, pour celui de Sainte-Victoire d'Arthabaska, l'évêque trouva judicieux de demander à la Congrégation de Notre-Dame qui était à Arthabaska depuis 1870. Les Sœurs de la Charité d'Ottawa se fixèrent à Saint-François-du-Lac et à Odanak, les Sœurs de la Présentation de Marie à Drummondville et à Saint-David, deux paroisses situées à proximité du diocèse de Saint-Hyacinthe où se trouvait leur noviciat.

\section{L'essaimage des Frères éducateurs}

Les congrégations masculines étaient largement moins répandues puisque, en 1904, seulement 5 maisons étaient sous leur direction : les Frères du Sacré-Cœur à Arthabaska et à Saint-Guillaume, et les Frères des Écoles chrétiennes, à Baie-du-Febvre, à Saint-Grégoire et à Nicolet.

\section{Les Frères du Sacré-Cour à Arthabaska}

C'est en 1872 que les Frères du Sacré-Cœur s'établirent à Arthabaska, devenant ainsi le premier institut masculin avec maison provinciale et noviciat, incorporé dans le diocèse. Mais, ce n'était pas leur premier lieu d'implantation en Amérique (1847) car ils arrivèrent à Mobile, en Alabama ${ }^{14}$. Toutefois, la maison-mère était localisée à Indianapolis. Ils cherchaient aussi à prendre racine au Canada. Mais, ils reçurent des réponses négatives des évêques de Montréal et de Toronto. Or, la demande d'enseignants religieux ne manquait pas. Ainsi, le curé de la paroisse de Saint-Christophe d'Arthabaska, Philippe-Hyppolyte Suzor, harcelait son évêque pour qu'il lui en trouve. En 1870, il cherchait des frères pour le collège commercial que la commission scolaire désirait établir dans la maison Wadleigh. Il commença par s'adresser aux Frères de Sainte-Croix qui déclinèrent son offre. Puis, voilà qu'une rumeur, répandue par le journal l'Union des Cantons de l'Est, disait que les prêtres du Séminaire de Nicolet allaient remplir la charge. Dès qu'ils eurent vent de la quête de Suzor, les Frères du SacréCour se montrèrent intéressés. Ils en firent part à l'évêque de Trois-Rivières qui s'empressa à son tour de communiquer cette information au curé d'Arthabaska. Il l'autorisa à leur faire connaître les conditions locales de leur résidence et de les assurer du bon accueil qu'il leur réserverait dans son diocèse.

Les frères acceptèrent et emménagèrent dans leur maison-école. L'amélioration des conditions matérielles du bâtiment exigea bientôt des

14. MARTEL, Jules, Histoire du collège de Victoriaville, tome I, Arthabaska 1872-1905, Victoriaville, 1965, 93 p. 
investissements que la congrégation ne pouvait apporter sans devenir propriétaire. Elle en avait l'occasion puisque la commission scolaire lui avait offert gratuitement le terrain et la maison, pourvu qu'elle construise un collège. Les commissaires évaluaient alors leur contribution à 4240 dollars. Le contrat renferma une clause de remboursement advenant le départ des frères. Ils acceptèrent l'entente qui, pour être valide, demandait l'incorporation civile. Même s'ils estimaient peu libérale et coûteuse la procédure de l'enregistrement de la charte, ils s'y plièrent, car c'était la seule voie ouverte au développement de la communauté. Ils l'obtinrent en 1875. Ils mirent cependant 17 ans à prendre racine dans une autre paroisse du diocèse de Nicolet. Comme les Frères des Écoles chrétiennes étaient déjà bien connus dans la région, ce fut plutôt à eux qu'on fit appel entre 1879 et 1888.

\section{La place des Frères des Écoles chrétiennes}

Le curé Didier Paradis avait aussi pour visée de faire venir les Frères des Écoles chrétiennes à Baie-du-Febvre. À nouveau partenaire de la fabrique, il partagea à moitié le prix d'achat d'un terrain et des bâtiments évalués 8000 dollars. C'est là que les frères résidèrent à leur arrivée en 1877. Peu après, les mêmes partenaires se divisèrent les coûts de la construction d'une aile de 6200 dollars. Les travaux débutèrent tôt en 1878 . L'étape suivante fut celle de la signature d'un contrat avec la commission scolaire locale car l'école des frères était soumise à sa juridiction ${ }^{15}$. Il fut passé au presbytère par les commissaires, les représentants de la fabrique et la congrégation, en présence du curé. L'acte renfermait les clauses qui doivent assurer le soutien matériel des frères : l'immeuble, la terre mise en exploitation, les revenus qui lui étaient attachés, la perception de la rétribution mensuelle par enfant. Les frères se voyaient également reconnaître le droit d'aliéner une partie de la propriété pourvu que les revenus fussent appliqués à l'œuvre. Remarquons que les frères n'obtenaient pas la propriété civile de ces biens. En effet, bien qu'incorporée en 1875, la congrégation n'avait encore en 1880 que peu de biens immobiliers en propre ${ }^{16}$.

Le contrat contenait cependant le germe de sa propre modification. Elle se produisit en 1886. À ce moment-là, le mécontentement était partout et avait plusieurs causes : la première résidait dans l'incompatibilité entre l'exploitation de la ferme et le charisme de la communauté, une autre dans la gestion de la propriété foncière par un frère directeur dont la compétence en la matière était fortement interrogée. S'ajoutaient des tracas de toutes sortes dont des sollicitations lassantes qui créaient une situation de crise, envenimée par la personnalité du frère directeur et une rumeur inquiétante sur la moralité d'un religieux. Bref, on en vint à proposer de

15. Archives des Frères des Écoles chrétiennes (AFEC), Laval, Dossier Baie-du-Febvre, " Acte passé devant le notaire Belcourt, à La Baie, 25 décembre 1877 ".

16. VoIsINe, Nive, Les Frères des Écoles chrétiennes au Canada, tome 1, La conquête de l'Amérique, 1837-1880, Sainte-Foy (Québec), Anne Sigier, 1987, p. 416-422. 
remplacer la congrégation. Une intervention extérieure à la paroisse semblait nécessaire. L'évêque du nouveau diocèse de Nicolet s'en mêla car il désirait que la communauté s'installe dans la ville épiscopale. Mais, il voyait bien que rien ne se ferait tant qu'une solution ne serait pas apportée aux problèmes similaires vécus à Baie-du-Febvre et à Saint-Grégoire.

Établis dans cette dernière paroisse depuis 1879, les frères habitaient une maison d'école neuve, construite par un groupe de citoyens au coût de 2700 dollars, sur un terrain acheté grâce à une substantielle contribution de 1600 dollars de la fabrique. Selon le curé, ce soutien financier s'était fait au détriment des investissements pourtant jugés nécessaires à apporter au presbytère. En 1879, les frères avaient accepté des conditions matérielles assez semblables à celles de la Baie-du-Febvre ${ }^{17}$. Les revenus reposaient principalement sur l'exploitation d'une ferme. Puis, un désaccord naquit à propos des dépenses engendrées par le chauffage de cette "belle mais grande maison d'école ». Les partis se renvoyaient la responsabilité de fournir le combustible. Les frères menacèrent même de quitter la paroisse. Sans doute existait-il d'autres points de litiges car, en 1883, l'évêque du diocèse, Louis-François Laflèche, jugea utile de s'adresser au provincial, le Frère Réticius. Il lui rappela que les paroissiens de Saint-Grégoire avaient consenti de lourds sacrifices pour obtenir leurs services; ils tenaient en estime leur enseignement et ils seraient désolés de les voir partir. Enfin, l'évêque le mettait en garde : «Soyez convaincu que l'abandon de cette fondation vous fera un tort considérable dans toute cette région ${ }^{18}$. "

Les Frères des Écoles chrétiennes ne quittèrent aucun des deux villages. Toutefois, ils obtinrent, les 13 avril et 7 juin 1886, une modification majeure aux contrats qui les liaient aux commissions scolaires et aux fabriques. Ils rétrocédaient leurs fermes et, de leur côté, les commissaires ajustaient en conséquence leurs salaires C'est ainsi que la fabrique de la Baie-du-Febvre reprit la terre. Elle la vendit. Puis, afin d'aider la commission scolaire à rencontrer ses nouvelles obligations, chaque année elle versa aux frères les intérêts du capital de la vente. Ces tractations reçurent évidemment l'aval de l'évêque. Intéressé à plus d'un point de vue, il délégua le curé de la paroisse cathédrale pour s'informer auprès des Frères visiteurs des conditions que poserait la communauté pour s'installer à Nicolet. Déterminé à les recevoir, le prélat avait fait acheter par la corporation épiscopale le bâtiment qui avait jadis abrité le collège-séminaire, et, à la satisfaction de la commission scolaire ${ }^{19}$, l'avait fait réparer. Il avait également fait l'achat d'un

17. AFEC, Laval, Dossier Saint-Grégoire, "Conditions requises pour l'érection de l'établissement des frères des Écoles Chrétiennes, dans la Paroisse de Saint-Grégoire ", Clause manuscrite, spécifique : "MM. les commisairres donneront aux Frères l'usage d'une maison et de quarante-cinq arpents de bonne terre, plus une somme annuelle de deux cent cinquante piastres, plus encore l'allocation spéciale et annuelle du gouvernement. Si l'allocation du Gouvt n'allait pas à cent piastres, MM. les commissaires y suppléreaient. "

18. AFEC, Laval, Laflèche au Cher Frère Réticius, 23 avril 1883.

19. Prudents, les frères firent inscrire une clause d'exclusivité disant qu'aucune corporation religieuse ou ecclésiastique ne devra établir un cours commercial à Nicolet. Nulle 
terrain adjacent. La corporation épiscopale, la commission scolaire et la congrégation passèrent un contrat le 27 août 1887. Le 13 mai 1891, une cession de l'épiscopat donna aux frères la pleine propriété des biens dont ils avaient eu l'usage depuis leur arrivée. De plus, l'évêque ajouta une somme de 700 dollars pour l'amélioration des classes. En somme, un succès dont le prélat pouvait se réjouir.

Devenu plus ouvert à la participation de l'institution ecclésiale à l'éducation, comme le démontrent diverses mesures législatives, le gouvernement du Bas-Canada la chargea de l'instruction publique. Rôle qu'elle n'avait pas la possibilité de remplir adéquatement. Néanmoins, à la suite de la loi de 1824, les fabriques avaient contribué à la création de nombreuses écoles de villages.

Le gouvernement prit en main ses responsabilités. Le succès des Écoles de syndics (1829) est principalement dû au financement gouvernemental, la preuve étant que la cessation des subventions, en 1836, entraîna la fermeture d'un très grand nombre d'écoles. La réorganisation scolaire (1841) qui suivit les Rébellions de 1837-1838, ne s'acheva qu'en 1845 et 1846 lorsque furent précisés les moyens de son financement. La Charte de l'éducation, car c'est ainsi qu'elle est appelée, était donnée. Elle ouvrait la porte à l'influence et à l'action de l'Église.

Proactive et non pas supplétive, elle mobilisa le clergé paroissial, les marguilliers de la fabrique et les communautés religieuses pour s'accaparer l'école de village. Les couvents et les académies qui s'ouvrirent dans le diocèse de Nicolet peuvent servir à illustrer le processus qui s'est mis en place partout au Québec. Il ne s'arrêta pas avec le siècle, bien au contraire. En effet, grâce à l'apport nouveau des communautés immigrantes, le mouvement s'amplifia et s'étendit à des diocèses moins touchés jusque-là, TroisRivières, par exemple.

crainte n'était justifiée tant les commissaires se montraient heureux des coûts moindres de l'enseignement religieux sur l'enseignement laïc : une économie estimée à 850 dollars en 1886-1887. 


\section{RÉSUMÉ}

L'établissement des couvents et des académies figure parmi les signes les plus évidents de l'emprise de l'Église sur l'enseignement dans le second $19^{e}$ siècle. Le législateur l'a rendu possible : il a favorisé les conditions matérielles des congrégations religieuses, autorisé la fabrique à investir en éducation et a accordé à l'Église une grande influence par sa loi sur l'Instruction publique. Néanmoins, ceci n'eut été rien sans la présence des communautés enseignantes, immigrantes ou autochtones, sans le dynamisme des curés, sans l'accord de la population ni sans la fabrique ici placée au cœur de la démonstration.

$$
\text { ABSTRACT }
$$

The establishing of convents and academies constitutes the most apparent sign of the Church's hold on education during the second half of the 19th Century. Legislation made it possible: it eased the material life of religious congregations, authorized the fabric to invest in education, and gave the Church a strong influence thanks to the Act on Public Instruction. However, this Act would have been powerless without the presence of teaching communities, local or foreign, without the dynamism of the priests, without the agreement of the population, and without the fabric, the latter being at the center of our demonstration. 